2019 4th International Conference on Information Technology, Information Systems and Electrical Engineering (ICITISEE)

EICITISEE

took place 20-21 November 2019 in Yogyakarta, Indonesia.

$\begin{array}{ll}\text { IEEE catalog number: } & \text { CFP19G48-ART } \\ \text { ISBN: } & 978-1-7281-5118-2\end{array}$

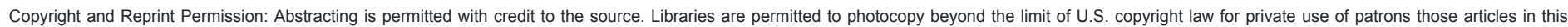

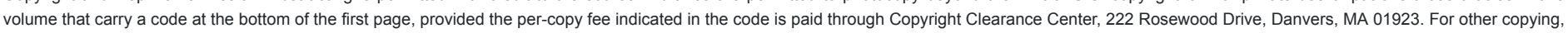
reprint or republication permission, write to IEEE Copyrights Manager, IEEE Operations Center, 445 Hoes Lane, Piscataway, NJ 08854 . All rights reserved. Copyright $\odot 2019$ by IEEE. 


\section{General Chair}

Kusrini Kusrini (AMIKOM Yogyakarta University, Indonesia)

\section{Publication Chair}

Sumarni Adi (Universitas AMIKOM Yogyakarta, Indonesia)

Akhmad Dahlan (Universitas Amikom Yogyakarta, Indonesia)

\section{Secretary}

Yoga Pristyanto (Universitas Amikom Yogyakarta, Indonesia)

Nila Puspitasari (Universitas AMIKOM Yogyakarta, Indonesia)

\section{Treasurer}

Sumarni Adi (Universitas AMIKOM Yogyakarta, Indonesia)

\section{Chair of Technical Program Committee}

Emha Taufiq Luthfi (Universitas AMIKOM Yogyakarta, Indonesia)

Lesnanto Multa Putranto (UGM, Indonesia)

Arief Setyanto (Universitas AMIKOM Yogyakarta, Indonesia)

Andi Sunyoto (Universitas AMIKOM Yogyakarta, Indonesia)

Ferry Wahyu Wibowo (Universitas Amikom Yogyakarta, Indonesia) 


\section{Technical Program Committee}

\begin{tabular}{|c|c|c|}
\hline Intan Ermahani A. Jalil & Universiti Teknikal Malaysia Melaka & Malaysia \\
\hline Mohd Helmy Abd Wahab & Universiti Tun Hussein Onn Malaysia & Malaysia \\
\hline MS. Hendriyawan Achmad & Yogyakarta University of Technology & Indonesia \\
\hline Sumarni Adi & Universitas AMIKOM Yogyakarta & Indonesia \\
\hline I Adiyasa & Gadjah Mada University & Indonesia \\
\hline I Made Agastya & Universitas Amikom Yogyakarta & Indonesia \\
\hline Mohd Khairul Ikhwan Ahmad & Universiti Tun Hussein Onn Malaysia & Malaysia \\
\hline Ali Qusay Al-Faris & Assumption College & USA \\
\hline Shajith Ali & SSN College of Engineering, Chennai & India \\
\hline Gede Angga Pradiptha & Institut Teknologi dan Bisnis STIKOM Bali & Indonesia \\
\hline Igi Ardiyanto & Universitas Gadjah Mada & Indonesia \\
\hline Dhani Ariatmanto & Universitas Amikom Yogyakarta & Indonesia \\
\hline Agus Aribowo & Universitas Pembangunan Nasional Veteran Yogyakarta & Indonesia \\
\hline Andria Arisal & Indonesian Institute of Sciences & Indonesia \\
\hline Arti Arya & PESIT-Bangalore South Campus & India \\
\hline Ika Astuti & Universitas Amikom Yogyakarta & Indonesia \\
\hline Hasyim Asyari & Universitas Muhammadiyah Surakarta & Indonesia \\
\hline Azizul Azizan & Universiti Teknologi Malaysia (UTM) & Malaysia \\
\hline Aslina Baharum & Universiti Malaysia Sabah & Malaysia \\
\hline Aashish Bardekar & Sipna College of Engineering and Technology, Amravati & India \\
\hline Agus Bejo & Universitas Gadjah Mada & Indonesia \\
\hline Adha Imam Cahyadi & Universitas Gadjah Mada & Indonesia \\
\hline Alessandro Carrega & CNIT & Italy \\
\hline Maria Chiara Caschera & CNR & Italy \\
\hline Selem Charfi & HD Technology & France \\
\hline Wichian Chutimaskul & King Mongkut's University of Technology Thonburi & Thailand \\
\hline Domenico Ciuonzo & University of Naples Federico II, IT & Italy \\
\hline Akhmad Dahlan & Universitas Amikom Yogyakarta & Indonesia \\
\hline
\end{tabular}




\begin{tabular}{|c|c|c|}
\hline Ni Ketut Dewi Ari Jayanti & Institute of Technology and Business STIKOM Bali & Indonesia \\
\hline Andi Wahju Rahardjo Emanuel & Universitas Atma Jaya Yogyakarta & Indonesia \\
\hline Ahmad Fajar & Bina Nusantara University & Indonesia \\
\hline Muhammad Faris & Universitas Gadjah Mada & Indonesia \\
\hline Hanif Fatta & Universitas AMIKOM Yogyakarta & Indonesia \\
\hline Anggit Ferdita Nugraha & Universitas AMIKOM Yogyakarta & Indonesia \\
\hline Alireza Ghasempour & ICT Faculty & USA \\
\hline Arifiyanto Hadinegoro & Amikom Universty Yogyakarta & Indonesia \\
\hline Byeong-jun Han & Soongsil University & Korea (South) \\
\hline Muh Hanafi & Universitas Amikom Yogyakarta & Indonesia \\
\hline Seng Hansun & Universitas Multimedia Nusantara & Indonesia \\
\hline Hartatik Hartatik & STMIK AMIKOM Yogyakarta & Indonesia \\
\hline Mardhiya Hayaty & Universitas AMIKOM Yogyakarta & Indonesia \\
\hline Purwono Hendradi & Universitas Muhammadiyah Magelang & Indonesia \\
\hline Roberto Carlos Herrera Lara & National Polytechnic School & Ecuador \\
\hline Indriana Hidayah & Universitas Gadjah Mada & Indonesia \\
\hline Tonny Hidayat & Universitas AMIKOM Yogyakarta & Indonesia \\
\hline Nor Hikmah & Universitas AMIKOM & Indonesia \\
\hline Danial Hooshyar & Korea University & Korea (South) \\
\hline Hozairi Hozairi & Islamic University of Madura & Indonesia \\
\hline Imelda Imelda & Universitas Budi Luhur & Indonesia \\
\hline Rini Indrayani & Universitas Amikom Yogyakarta & Indonesia \\
\hline Nurulisma Ismail & Universiti Malaysia Perlis & Malaysia \\
\hline Iswandi Iswandi & Gadjah Mada University & Indonesia \\
\hline Ramkumar Jaganathan & VLB Janakiammal College of Arts and Science & India \\
\hline Arihant Jain & Jaipur Engineering College \& Research Centre & India \\
\hline Biao Jiang & The City University of New York & USA \\
\hline Dimitrios Kallergis & University of West Attica & Greece \\
\hline Sandy Kosasi & STMIK Pontianak & Indonesia \\
\hline
\end{tabular}




\begin{tabular}{|c|c|c|}
\hline Krisnawati Krisnawati & University of AMIKOM Yogyakarta & Indonesia \\
\hline Ryan Kristianto & Universitas Amikom Yogyakarta & Indonesia \\
\hline Sumit Kushwaha & KNIT Sultanpur & India \\
\hline Kusnawi Kusnawi & AMIKOM University & Indonesia \\
\hline Kusrini Kusrini & AMIKOM Yogyakarta University & Indonesia \\
\hline Pavel Loskot & Swansea University & United Kingdom (Great Britain) \\
\hline Emha Taufiq Luthfi & Universitas AMIKOM Yogyakarta & Indonesia \\
\hline Mahdin Mahboob & Stony Brook University & USA \\
\hline Robert Marco & STMIK Amikom Yogyakarta & Indonesia \\
\hline Prita Dewi Mariyam & Universitas Indonesia & Indonesia \\
\hline Ahlihi Masruro & Universitas AMIKOM Yogyakarta & Indonesia \\
\hline Ratheesh Kumar Meleppat & University of California Davis & USA \\
\hline Ahmed Mobashsher & The University of Queensland & Australia \\
\hline Seyed Sahand Mohammadi Ziabari & Vrije University of Amsterdam & The Netherlands \\
\hline Amrit Mukherjee & Jiangsu University & China \\
\hline I Wayan Mustika & Universitas Gadjah Mada & Indonesia \\
\hline Syibrah Naim & Universiti Sains Malaysia & Malaysia \\
\hline Warsun Najib & Universitas Gadjah Mada & Indonesia \\
\hline Asro Nasiri & University of Amikom Yogyakarta & Indonesia \\
\hline Shah Nazir & University of Peshawar & Pakistan \\
\hline Ruzelita Ngadiran & Universiti Malaysia Perlis & Malaysia \\
\hline Bhanu Nugraha & Universitas Amikom Yogyakarta & Indonesia \\
\hline Hanung Nugroho & Universitas Gadjah Mada & Indonesia \\
\hline Prapto Nugroho & Universitas Gadjah Mada & Indonesia \\
\hline Nitish Ojha & DIT University, Dehradun & India \\
\hline Ilker Ali Ozkan & Selcuk University & Turkey \\
\hline Oktavia Permata & Institut Teknologi Telkom Surabaya & Indonesia \\
\hline Anugerah Persada & Universitas Gadjah Mada & Indonesia \\
\hline Kiran Sree Pokkuluri & Shri Vishnu Engineering College for Women & India \\
\hline
\end{tabular}




\begin{tabular}{|c|c|c|}
\hline N. Prabaharan & SASTRA Deemed University & India \\
\hline Gede Pramudya Ananta & Universiti Teknikal Malaysia Melaka & Malaysia \\
\hline Andri Pranolo & Universitas Ahmad Dahlan & Indonesia \\
\hline Prihandoko Prihandoko & University of Gunadarma & Indonesia \\
\hline Yoga Pristyanto & Universitas Amikom Yogyakarta & Indonesia \\
\hline Reza Pulungan & Universitas Gadjah Mada & Indonesia \\
\hline Nila Puspitasari & Universitas AMIKOM Yogyakarta & Indonesia \\
\hline Guntur Putra & University of New South Wales & Australia \\
\hline Lesnanto Multa Putranto & UGM & Indonesia \\
\hline Ali Rafiei & University of Technology Sydney & Australia \\
\hline Suwanto Raharjo & Informatics of The Institut Sains \& Teknologi AKPRIND Yogyakarta & Indonesia \\
\hline Hemant Kumar Rath & Tata Consultancy Services & India \\
\hline Rizky Rizky & Universitas AMIKOM Yogyakarta & Indonesia \\
\hline Rumini Rumini & Universitas AMIKOM Yogyakarta & Indonesia \\
\hline G. p. Sajeev & Amrita Vishwa Vidyapeetham & India \\
\hline Andreas Sandiwan & Gadjah Mada University & Indonesia \\
\hline Banu Santoso & Universitas AMIKOM Yogyakarta & Indonesia \\
\hline Leo Santoso & Petra Christian University & Indonesia \\
\hline Sayantam Sarkar & Vijaya Vittala Institute of Technology & India \\
\hline Theopilus Bayu Sasongko & Universitas AMIKOM Yogyakarta & Indonesia \\
\hline Mithileysh Sathiyanarayanan & City, University of London & United Kingdom (Great Britain) \\
\hline Vaibhav Saundarmal & Marathwada Institute of Technology, Aurangabad & India \\
\hline Enny Sela & Universitas Teknologi Yogyakarta & Indonesia \\
\hline Erni Seniwati & Universitas AMIKOM Yogyakarta & Indonesia \\
\hline Anindita Septiarini & Univeristas Mulawarman & Indonesia \\
\hline Amel Serrat & USTO MB & Algeria \\
\hline Bayu Setiaji & Universitas AMIKOM Yogyakarta & Indonesia \\
\hline Noor Akhmad Setiawan & Universitas Gadjah Mada & Indonesia \\
\hline Emy Setyaningsih & Institute of Science \& Technology AKPRIND & Indonesia \\
\hline
\end{tabular}




\begin{tabular}{|c|c|c|}
\hline Arief Setyanto & Universitas AMIKOM Yogyakarta & Indonesia \\
\hline Iwan Setyawan & Satya Wacana Christian University & Indonesia \\
\hline Andik Setyono & Universitas Dian Nuswantoro & Indonesia \\
\hline Aditi Sharma & Quantum University, Roorkee, Uttarakhand & India \\
\hline Vesh Raj Sharma Banjade & Intel Corporation & USA \\
\hline Abdul Samad Shibghatullah & UCSI University & Malaysia \\
\hline Monika Sikri & Cisco Systems India Pvt Ltd. & India \\
\hline Amando Singun & Higher College of Technology, Muscat & Oman \\
\hline Achmad Solichin & Universitas Budi Luhur & Indonesia \\
\hline lickho Song & Korea Advanced Institute of Science and Technology & Korea (South) \\
\hline Yi-Jen Su & Shu-Te University & Taiwan \\
\hline Suhirman Suhirman & Universitas Teknologi Yogyakarta & Indonesia \\
\hline Andi Sunyoto & Universitas AMIKOM Yogyakarta & Indonesia \\
\hline Sutarman Sutarman & Faculty Of Information Technology and Business, University Technoloy of Yogyakarta & Indonesia \\
\hline Melwin Syafrizal & Universitas AMIKOM Yogyakarta & Indonesia \\
\hline Srinivasulu Tadisetty & Kakatiya University College of Engineering and Technology & India \\
\hline Chakib Taybi & Mohammed First University & Morocco \\
\hline Ivanna Timotius & Satya Wacana Christian University & Indonesia \\
\hline Evi Triandini & Institut Teknologi dan Bisnis STIKOM Bali & Indonesia \\
\hline Hastari Utama & Universitas Amikom Yogyakarta & Indonesia \\
\hline Ema Utami & Universitas Amikom Yogyakarta & Indonesia \\
\hline Dario Vieira & EFREI & France \\
\hline Anik Vitianingsih & Universitas Dr Soetomo & Indonesia \\
\hline Mochammad Wahyudi & Universitas Gadjah Mada & Indonesia \\
\hline Sri Ngudi Wahyuni & Universitas AMIKOM Yogyakarta & Indonesia \\
\hline Fikri Waskito & Universitas Gadjah Mada & Indonesia \\
\hline Leong Wen Chek & University of Malaya & Malaysia \\
\hline Sunu Wibirama & Universitas Gadjah Mada & Indonesia \\
\hline Adi Wibowo & Diponegoro University & Indonesia \\
\hline
\end{tabular}




\begin{tabular}{|c|c|c|}
\hline Ferry Wahyu Wibowo & Universitas Amikom Yogyakarta & Indonesia \\
\hline Sigit Wibowo & Gadjah Mada University & Indonesia \\
\hline Teguh Wibowo & Gadjah Mada University & Indonesia \\
\hline Ainul Yaqin & Universitas Amikom Yogyakarta & Indonesia \\
\hline Thaweesak Yingthawornsuk & King Mongkut's University of Technology Thonburi & Thailand \\
\hline Uky Yudatama & Universitas Indonesia & Indonesia \\
\hline Go Yun II & Heriot-Watt University Malaysia & Malaysia \\
\hline Muhammad Yusuf & University of Trunojoyo, Madura & Indonesia \\
\hline Sri Zuliana & UIN Sunan Kalijaga & Indonesia \\
\hline Nur Zareen Zulkarnain & Universiti Teknikal Malaysia Melaka & Malaysia \\
\hline
\end{tabular}




\section{Additional Reviewers}

\begin{tabular}{|c|c|c|}
\hline Intan Ermahani A. Jalil & Universiti Teknikal Malaysia Melaka & Malaysia \\
\hline Mohd Helmy Abd Wahab & Universiti Tun Hussein Onn Malaysia & Malaysia \\
\hline MS. Hendriyawan Achmad & Yogyakarta University of Technology & Indonesia \\
\hline I Wayan Adiyasa & Gadjah Mada University & Indonesia \\
\hline Ali Qusay Al-Faris & Assumption College & USA \\
\hline Shajith Ali & SSN College of Engineering, Chennai & India \\
\hline Gede Angga Pradiptha & Institut Teknologi dan Bisnis STIKOM Bali & Indonesia \\
\hline Dhani Ariatmanto & Universitas Amikom Yogyakarta & Indonesia \\
\hline Agus Sasmito Aribowo & Universitas Pembangunan Nasional Veteran Yogyakarta & Indonesia \\
\hline Andria Arisal & Indonesian Institute of Sciences & Indonesia \\
\hline Arti Arya & PESIT-Bangalore South Campus & India \\
\hline Aslina Baharum & Universiti Malaysia Sabah & Malaysia \\
\hline Maria Chiara Caschera & CNR & Italy \\
\hline Wichian Chutimaskul & King Mongkut's University of Technology Thonburi & Thailand \\
\hline Domenico Ciuonzo & University of Naples Federico II, IT & Italy \\
\hline Ni Ketut Dewi Ari Jayanti & Institute of Technology and Business STIKOM Bali & Indonesia \\
\hline Andi Wahju Rahardjo Emanuel & Universitas Atma Jaya Yogyakarta & Indonesia \\
\hline Ahmad Nurul Fajar & Bina Nusantara University & Indonesia \\
\hline Anggit Ferdita Nugraha & Universitas AMIKOM Yogyakarta & Indonesia \\
\hline Alireza Ghasempour & ICT Faculty & USA \\
\hline Seng Hansun & Universitas Multimedia Nusantara & Indonesia \\
\hline Hartatik Hartatik & STMIK AMIKOM Yogyakarta & Indonesia \\
\hline Mardhiya Hayaty & Universitas AMIKOM Yogyakarta & Indonesia \\
\hline Roberto Carlos Herrera Lara & National Polytechnic School & Ecuador \\
\hline Tonny Hidayat & Universitas AMIKOM Yogyakarta & Indonesia \\
\hline Danial Hooshyar & Korea University & Korea (South) \\
\hline Hozairi Hozairi & Islamic University of Madura & Indonesia \\
\hline Rini Indrayani & Universitas Amikom Yogyakarta & Indonesia \\
\hline Nurulisma Ismail & Universiti Malaysia Perlis & Malaysia \\
\hline Ramkumar Jaganathan & VLB Janakiammal College of Arts and Science & India \\
\hline Biao Jiang & The City University of New York & USA \\
\hline Sandy Kosasi & STMIK Pontianak & Indonesia \\
\hline Krisnawati Krisnawati & University of AMIKOM Yogyakarta & Indonesia \\
\hline Ryan Putranda Kristianto & Universitas Amikom Yogyakarta & Indonesia \\
\hline Sumit Kushwaha & KNIT Sultanpur & India \\
\hline Pavel Loskot & Swansea University & United Kingdom (Great Britain) \\
\hline Prita Dewi Mariyam & Universitas Indonesia & Indonesia \\
\hline Ratheesh Kumar Meleppat & University of California Davis & USA \\
\hline Amrit Mukherjee & Jiangsu University & China \\
\hline Syibrah Naim & Universiti Sains Malaysia & Malaysia \\
\hline Asro Nasiri & University of Amikom Yogyakarta & Indonesia \\
\hline Shah Nazir & University of Peshawar & Pakistan \\
\hline Ruzelita Ngadiran & Universiti Malaysia Perlis & Malaysia \\
\hline Hanung Adi Nugroho & Universitas Gadjah Mada & Indonesia \\
\hline Prapto Nugroho & Universitas Gadjah Mada & Indonesia \\
\hline Ilker Ali Ozkan & Selcuk University & Turkey \\
\hline Oktavia Ayu Permata & Institut Teknologi Telkom Surabaya & Indonesia \\
\hline Gede Pramudya Ananta & Universiti Teknikal Malaysia Melaka & Malaysia \\
\hline Prihandoko Prihandoko & University of Gunadarma & Indonesia \\
\hline Guntur Dharma Putra & University of New South Wales & Australia \\
\hline Lesnanto Multa Putranto & UGM & Indonesia \\
\hline Suwanto Raharjo & Informatics of The Institut Sains \& Teknologi AKPRIND Yogyakarta & Indonesia \\
\hline Bambang Riyono & Gadjah Mada University & Indonesia \\
\hline Leo Santoso & Petra Christian University & Indonesia \\
\hline Vaibhav Dudhaji Saundarmal & Marathwada Institute of Technology, Aurangabad & India \\
\hline Enny Sela & Universitas Teknologi Yogyakarta & Indonesia \\
\hline Ririn Septrisulviani & Universitas Gadjah Mada & Indonesia \\
\hline Amel Serrat & USTO MB & Algeria \\
\hline
\end{tabular}




\begin{tabular}{|l|l|l|}
\hline Lilis Susanti Setianingsih & Universitas Gadjah Mada & Indonesia \\
\hline Noor Akhmad Setiawan & Universitas Gadjah Mada & Indonesia \\
\hline Iwan Setyawan & Satya Wacana Christian University & Indonesia \\
\hline Abdul Samad Shibghatullah & UCSI University & Malaysia \\
\hline Achmad Solichin & Universitas Budi Luhur & Indonesia \\
\hline lickho Song & Korea Advanced Institute of Science and Technology & Korea (South) \\
\hline Andi Sunyoto & Universitas AMIKOM Yogyakarta & Indonesia \\
\hline Melwin Syafrizal & Universitas AMIKOM Yogyakarta & Indonesia \\
\hline Srinivasulu Tadisetty & Kakatiya University College of Engineering and Technology & India \\
\hline Chakib Taybi & Mohammed First University & Morocco \\
\hline Ivanna Timotius & Satya Wacana Christian University & Indonesia \\
\hline Evi Triandini & Institut Teknologi dan Bisnis STIKOM Bali & Indonesia \\
\hline Anik Vega Vitianingsih & Universitas Dr Soetomo & Indonesia \\
\hline Leong Wen Chek & University of Malaya & Malaysia \\
\hline Adi Wibowo & Diponegoro University & Indonesia \\
\hline Ainul Yaqin & Universitas Amikom Yogyakarta & Indonesia \\
\hline Thaweesak Yingthawornsuk & King Mongkut's University of Technology Thonburi & Thailand \\
\hline Uky Yudatama & Universitas Indonesia & Indonesia \\
\hline Sri Utami Zuliana & UIN Sunan Kalijaga & Indonesia \\
\hline
\end{tabular}




\section{4th International Conference on Information Technology, Information Systems and Electrical Engineering (ICITISEE)}

Parallel Session 1-A

Evaluation of Golomb Ruler Optimum Performance for NG-PON2 Networks

Satrio Priambodo (Telkom University, Indonesia), Brian Pamukti (Telkom University, Indonesia), Akhmad Hambali (Telkom University, Indonesia)

Entropy and Information Gain Analysis on Low Cost BCI for Motorbike Users to Prevent Accident

Rolly Maulana Awangga (Institut Teknologi Bandung \& Politeknik Pos Indonesia, Indonesia), Syafrial Fachri Pane (Applied Bachelor Program of Informatics Engineering, Politeknik Pos Indonesia, Indonesia), Dinda Majesty (Politeknik Pos Indonesia, Indonesia), Moch Yusuf Asyhari (Universitas Islam Indonesia, Indonesia)

Study on C-Band Electromagnetic Wave Absorber made of S-Ring Resonator

Farhan Fathir Lanang (Telkom University, Indonesia), Levy Olivia Nur (Telkom University, Indonesia), Budi Syihabuddin (Telkom University, Indonesia), Bambang Setia Nugroho (Telkom University, Indonesia), Agus D. Prasetyo (Telkom University, Indonesia), Heroe Wijanto (Telkom University, Indonesia)

Performance evaluation of M-ary modulated DCO-OFDM in an Indoor Visible Light Communication System

Nurul Fatma Milia (Telkom University, Indonesia), Erna Sugesti (Telkom University, Indonesia), Desti Madya Saputri (Telkom University, Indonesia), Brian Pamukti (Telkom University, Indonesia)

Impact Analysis of Location and Penetration Level of DFIG on Small Signal Stability of Power System

Avrin Nur Widiastuti (UGM, Indonesia), Sarjiya Sarjiya (Gadjah Mada University, Indonesia), Sasongko Hadi (Universitas Gadjah Mada, Indonesia)

Analysis of Transient Signal using Hilbert-Huang Transform for Chatter Monitoring in Turning Process

Agus Susanto (State Polytechnic of Madiun, Indonesia), Keiji Yamada (Hiroshima University, Japan), Ryutaro Tanaka (Hiroshima University, Japan), Muizuddin Azka (Agency for the Assessment and Application of Technology (BPPT), Indonesia), Katsuhiko Sekiya (Hiroshima University, Japan), Murman Dwi Prasetio (Telkom University, Indonesia), Putri Novia (State Polytechnic of Madiun, Indonesia)

Parallel Session 1-B

Automatic Cacao Pod Detection Under Outdoor Condition Using Computer Vision

Yulia Ekawaty (Universitas Hasanuddin, Indonesia), Indrabayu A (Hasanuddin University, Indonesia), Intan Sari Areni (Hasanuddin University, Indonesia)

Using Big Data and Al to Examine Product Engagement in Social Media Influencer Posts

Stuart Barnes (King's College London, United Kingdom (Great Britain)), Richard Rutter (Australian College of Kuwait, United Kingdom (Great Britain))

The Best Parameters to Select Instagram Account for Endorsement using Web Scraping

Muhammad Ichwandar Akrianto (Universitas Amikom Yogyakarta, Indonesia), Anggit Dwi Hartanto (Universitas Amikom Yogyakarta,

Indonesia), Adri Priadana (Universitas Jenderal Achmad Yani Yogyakarta, Indonesia)

Decision Support System for Boarding house Search Using Topsis Algorithm

Ainul Yaqin (Universitas Amikom Yogyakarta, Indonesia), Akhmad Dahlan (Universitas Amikom Yogyakarta, Indonesia), Tonny Hidayat (Universitas AMIKOM Yogyakarta, Indonesia), Reza Mardiansyah Putra (Amikom, Indonesia)

The Selection of Periodic Salary Increment of Civil Servants using Fuzzy MADM

Wahyuni Eka Sari (Politeknik Pertanian Negeri Samarinda, Indonesia), Silmi Fauziati (Universitas Gadjah Mada, Indonesia)

Time-Frequency Analysis (TFA) method for load identification on Non-Intrusive Load Monitoring

Nur Iksan (Universitas Negeri Semarang, Indonesia), Erika Devi Udayanti (Universitas Dian Nuswantoro, Indonesia)

Parallel Session 1-C

Automatically Regulates Non Player Character Behavior Using Fuzzy Logic As An Artificial Intelligence Mechanism For Action Makers Tonny Hidayat (Universitas AMIKOM Yogyakarta, Indonesia), Ika Astuti (Universitas Amikom Yogyakarta, Indonesia), Akhmad Dahlan (Universitas Amikom Yogyakarta, Indonesia)

Comparison Analysis of the Implementation of the AHP and AHP-PROMETHEE Methods for the Selection of Trainees

Dewi Anisa Istiqomah (Universitas AMIKOM Yogyakarta, Indonesia), Vikky Aprelia (Universitas AMIKOM Yogyakarta, Indonesia)

Clustering K Means for Criteria Weighting With Improvement Result of Alternative Decisions Using SAW and TOPSIS

Erna Daniati (Indonesia \& Universitas Nusantara PGRI Kediri, Indonesia), Hastari Utama (Universitas Amikom Yogyakarta, Indonesia)

Animal metamorphosis learning media using android- Based augmented reality technology

Agus Purwanto (Lecturer of University Amikom Yogyakarta \& CREATIVE DIRECTOR OF PARAMA CREATIVE, Indonesia), Mei Parwanto Kurniawan (University of AMIKOM Yogyakarta, Indonesia), Ahmad Zaid Rahman (Universitas AMIKOM Yogyakarta, Indonesia)

Augmented Reality of Android-Based Learning Media of Sun and Earth Structure

Mei Parwanto Kurniawan (University of AMIKOM Yogyakarta, Indonesia), Agus Purwanto (Lecturer of University Amikom Yogyakarta \& CREATIVE DIRECTOR OF PARAMA CREATIVE, Indonesia), Muhammad Fahmi Mansur (University of Amikom Yogyakarta, Indonesia) 
Implementation Least Means Square Algorithm for Real-Time Active Noise Cancellation on FPGA

Muhammad Nur Shahreen Osman (Universiti Teknologi MARA, Malaysia), Ili Shairah Abdul Halim (Universiti Teknologi MARA \& Faculty of

Electrical Engineering, Malaysia), Siti Lailatul Mohd Hassan (Universiti Teknologi Mara, Malaysia), A'zraa Afhzan Ab Rahim (Universiti

Teknologi MARA, Malaysia), Noor Ezan Abdullah (Universiti Teknologi Mara, Malaysia)

\section{Parallel Session 1-D}

Comparison of Pornographic Image Classification based on Texture, Color, and Shape Features

I Wayan Pandu Swardiana (Universitas AMIKOM Yogyakarta, Indonesia), Arief Setyanto (Universitas AMIKOM Yogyakarta, Indonesia), Sudarmawan Sudarmawan (AMIKOM Yogyakarta University, Indonesia)

Implementation of 2DPCA and SOM Algorithms to Determine Sex According to Lip Shapes

Nor Hikmah (Universitas AMIKOM, Indonesia)

Comparison of Scale Invariant Feature Transform and Speed Up Robust Feature for Image Forgery Detection Copy Move

Reflan Nuari (Amikom University Yogyakarta, Indonesia), Ema Utami (Universitas Amikom Yogyakarta, Indonesia), Suwanto Raharjo (Informatics of The Institut Sains \& Teknologi AKPRIND Yogyakarta, Indonesia)

Potential Detection of Lentigo Maligna Melanoma on Solar Lentigines Image Based on Android

Casi Setianingsih (Telkom University, Indonesia)

Personality Features Identification from Handwriting Using Convolutional Neural Networks

Sri Fatimah (Universitas Jenderal Achmad Yani, Indonesia), Esmeralda Contessa Djamal (Universitas Jenderal Achmad Yani, Indonesia),

Faiza Renaldi (Universitas Jenderal Achmad Yani, Indonesia)

Comparison of Naive Bayes and K-NN method on Tuition Fee Payment Overdue Prediction

Kusrini Kusrini (AMIKOM Yogyakarta University, Indonesia), Emha Taufiq Luthfi (Universitas AMIKOM Yogyakarta, Indonesia), Muqorobin

Muqorobin (Universitas AMIKOM Yogyakarta, Indonesia), Robi Abdullah (Universitas AMIKOM Yogyakarta, Indonesia)

\section{Parallel Session 1-E}

Impact of Device Orientation for Visible Light Communication in Closed Room

Amirullah Wijayanto (Telkom University, Indonesia), Kris Sujatmoko (Telkom University, Indonesia), Brian Pamukti (Telkom University, Indonesia)

Evaluate Number of LED on Reflector Room for Optical Wireless Communication

Dyndra Ramadhanti (Telkom University, Indonesia), Brian Pamukti (Telkom University, Indonesia), Kris Sujatmoko (Telkom University, Indonesia)

Blockchain-based Secure Data Storage for Door Lock System

Ulfah Nadiya (Indonesian Institute of Science, Indonesia), Muhammad Ilham Rizqyawan (Indonesian Institute of Sciences, Indonesia), Oka Mahendra (Indonesian Institute of Sciences, Indonesia)

Detection System for Cigarette Smoke

Junaidy B Sanger (Universitas Katolik De La Salle Manado, Indonesia), Lanny Sitanayah (Universitas Katolik De La Salle Manado, Indonesia), Vivie D. Kumenap (Universitas Katolik De La Salle Manado, Indonesia)

A Novel Approach to Resource Starvation Attacks on MQTT Brokers

Ricardo Da Paz (Edith Cowan University, Australia), Aiden Sehovic (Edith Cowan University, Australia), David Cook (Edith Cowan University, Australia), Leisa J Armstrong (Edith Cowan University, Australia)

Design and Implementation of Node Gateway with MQTT and CoAP Protocol for IoT Applications

Ahmad Zainudin (Politeknik Elektronika Negeri Surabaya, Indonesia), Mohamad Fahmi Syaifudin (PENS, Indonesia), Nanang Syahroni (Politeknik Elektronika Negeri Surabaya, Indonesia)

Parallel Session 2-A

Electroencephalograph Recording with Ten-Twenty Electrode System Based on Arduino Mega 2560 Titis Bagus Kurnianadi (Soegijapranata Catholic University, Indonesia), Florentinus Budi Setiawan (Soegijapranata Catholic University, Indonesia)

Diminish the Peak Value of the Cogging Torque by Modifying of the Stator Teeth Tip Geometry

Herlina Wahab (Sriwijaya University \& University of Indonesia, Indonesia), Rudy Setiabudy (Universitas Indonesia (UI), Indonesia), Syamsuri Zaini (Sriwijaya University, Indonesia)

Level Crossing Rate Impact on Routing Performance in Adhoc Networks for Device-to-Device Communication

Istikmal Istikmal (Telkom University, Indonesia), Edwar Edwar (Telkom University, Indonesia)

An Adaptive Scaling Factor for Multiple Watermarking Scheme

Dhani Ariatmanto (Universitas Amikom Yogyakarta, Indonesia), Ferda Ernawan (Universiti Malaysia Pahang, Malaysia) 
Development of Internet-of-Things based Building Monitoring System for Supporting the Disaster Mitigation in The City

Asep Najmurrokhman (Universitas Jenderal Achmad Yani, Indonesia), Kusnandar Kusnandar, k (UNJANI \& UNJANI, Indonesia), Udin Komarudin (Universitas Jenderal Achmad Yani, Indonesia), Ahmad Daelami (Universitas Jenderal Achmad Yani, Indonesia), Restu Arisandy (Universitas Jenderal Achmad Yani, Indonesia)

MQTT Performance as a Message Protocol in an loT based Chili Crops Greenhouse Prototyping

Dania Eridani (Diponegoro University, Indonesia), Kurniawan Martono (Diponegoro University, Indonesia)

Development of Automatic Waste Segregator with Monitoring System

A'zraa Afhzan Ab Rahim (Universiti Teknologi MARA, Malaysia), Nurisha Hania Kamarudin (UiTM, Malaysia), Noor Ezan Abdullah (Universiti Teknologi Mara, Malaysia), Ili Shairah Abdul Halim (Universiti Teknologi MARA \& Faculty of Electrical Engineering, Malaysia), Siti Lailatul Mohd Hassan (Universiti Teknologi Mara, Malaysia)

Quantization Effect on 5 G Millimeter Wave Communication

Nasrullah Armi (Indonesian Institute of Sciences \& Telkom University, Indonesia), Chaeriah Bin Ali Wael (Indonesian Institute of Sciences, Indonesia), Arumjeni Mitayani (Indonesian Institute of Sciences, Indonesia), Arief Suryadi Satyawan (Waseda University, Japan \& Indonesian Institute of Sciences, Indonesia), Galih Nugraha Nurkahfi (Indonesian Institute of Science(LIPI), Indonesia)

\section{Parallel Session 2-B}

Human Perception Evaluation toward End of File Steganography Method's Implementation Using Multimedia File (Image, Audio, and Video) Rini Indrayani (Universitas Amikom Yogyakarta, Indonesia)

Hybrid Encryption Technique using Cyclic Bit Shift and RC4

Dina Evita Sari (Dian Nuswantoro University, Indonesia), Happy Niti Noor Muchsin (Dian Nuswantoro University, Indonesia), De Rosal Ignatius Moses Setiadi (Dian Nuswantoro University, Indonesia), Christy Atika Sari (Dian Nuswantoro University, Indonesia), Eko Hari Rachmawanto (Dian Nuswantoro University, Indonesia)

Steganographic-Algorithm and Length Estimation Classification on MP3 Steganalysis with Convolutional Neural Network Muhammad Rizki Duwinanto (Bandung Institute of Technology, Indonesia), Rinaldi Munir (Institut Teknologi Bandung, Indonesia)

QIM-based Audio Watermarking using Polar-based Singular Value in DCT Domain

Gelar Budiman (Telkom University \& Institute Technology Bandung, Indonesia), Ledya Novamizanti (Telkom University, Indonesia), Allwinnaldo Allwinnaldo (Telkom University, Indonesia)

Dual Encryption Method for File Security

Daniel Mahardika Yusuf (Dian Nuswantoro University, Indonesia), De Rosal Ignatius Moses Setiadi (Dian Nuswantoro University, Indonesia) Christy Atika Sari (Dian Nuswantoro University, Indonesia), Eko Hari Rachmawanto (Dian Nuswantoro University, Indonesia), Rabei Raad Al (Universiti Tun Hussein Onn Malaysia, Malaysia)

Design of Blockchain-Based Electronic Election System Using Hyperledger: Case of Indonesia

Donny Seftyanto (Badan Siber dan Sandi Negara, Indonesia), Amiruddin Amiruddin (Sekolah Tinggi Sandi Negara \& Badan Siber dan Sandi Negara, Indonesia), Arif Rahman Hakim (Sekolah Tinggi Sandi Negara, Indonesia)

Mitigation of Cryptojacking Attacks Using Taint Analysis

Arief Dwi Yulianto (Telkom University, Indonesia), Muhammad Al Makky (Telkom University, Indonesia), Parman Sukarno (Telkom University, Indonesia), Aulia Arif Wardana (Telkom University, Indonesia)

User Satisfaction Levels Sentiment Analysis Toward Goods Delivery Service On Twitter Using Support Vector Machine Algorithm (SVM)

Andia Enggar Mayasari (Universitas AMIKOM Yogyakarta, Indonesia), Anggit Dwi Hartanto (Universitas Amikom Yogyakarta, Indonesia)

Parallel Session 2-C

Planar Dipole MIMO Array Antenna for Mobile Robot Communications at $5.6 \mathrm{GHz}$

Muhsin Muhsin (Institut Teknologi Telkom Surabaya, Indonesia)

Natural Disaster Application on Big Data and Machine Learning: A Review

Rania Arinta (Universitas Atma Jaya Yogyakarta, Indonesia), Andi Wahju Rahardjo Emanuel (Universitas Atma Jaya Yogyakarta, Indonesia)

Machine Learning Classifiers for Autism Spectrum Disorder: Review

Dadang Eman (Universitas Atma Jaya Yogyakarta, Indonesia), Andi Wahju Rahardjo Emanuel (Universitas Atma Jaya Yogyakarta, Indonesia)

Comparison of Classification Methods using Historical Loan Application Data

Yohanes R. Laberto Kelen (Universitas Atma Jaya Yogyakarta, Indonesia), Andi Wahju Rahardjo Emanuel (Universitas Atma Jaya Yogyakarta, Indonesia)

The Undersampling Effects on RANDSHUFF Oversampling Algorithms

Tora Fahrudin (University of Telkom, Indonesia)

Meta-Algorithms for Improving Classification Performance in the Web-phishing Detection Process

Anggit Ferdita Nugraha (Universitas AMIKOM Yogyakarta, Indonesia), Luthfia Rahman (Universitas Gadjah Mada, Indonesia)

The Prototype of Decision Support System For Selecting The Lands of Crops

Dema Mathias Lumban Tobing (Universitas Amikom Yogyakarta, Indonesia), Julia Kurniasih (Amikom University, Indonesia), Yulius Nahak Tetik (Amikom University, Indonesia), Kusrini Kusrini (AMIKOM Yogyakarta University, Indonesia) 
Comparison Of SIFT and SURF Methods For Porn Image Detection

Hartatik Hartatik (STMIK AMIKOM Yogyakarta, Indonesia), Arief Setyanto (Universitas AMIKOM Yogyakarta, Indonesia), Kusrini Kusrini

(AMIKOM Yogyakarta University, Indonesia), I Made Artha Agastya (Universitas Amikom Yogyakarta, Indonesia)

Parallel Session 2-D

Self-Organizing Map (SOM) For Diagnosis Coronary Heart Disease

Triyanna Widiyaningtyas (Universitas Negeri Malang, Indonesia), Ilham A. E. Zaeni (State University of Malang, Indonesia), Putri Wahyuningrum (Universitas Negeri Malang, Indonesia)

Effect of Giving N Value on ADASYN-N Method for Classification of Imbalanced Nominal Data

Sri Rahayu (Universitas Janabadra, Indonesia), Jeffry Andhika Putra (Universitas Janabadra, Indonesia), Yumarlin MZ (Universitas Janabadra, Indonesia)

Sentiment Analysis in Airline Tweets Using Mutual Information for Feature Selection

Hastari Utama (Universitas Amikom Yogyakarta, Indonesia)

Optimization Of Parameter Support Vector Machine (SVM) using Genetic Algorithm to Review GO-JEK Services

Windha Mega Pradnya Dhuhita (Universitas AMIKOM Yogyakarta, Indonesia), Haryoko Haryoko (University of AMIKOM Yogyakarta,

Indonesia)

A Review of Long Short-Term Memory Method for Hate Speech Classification on Twitter

Syahrul Syafaat Syam (Telkom University, Indonesia), Budhi Irawan (Telkom University, Indonesia), Casi Setianingsih (Telkom University, Indonesia)

Comparison Performance of Decision Tree Classification Model for Spam Filtering with or without the Recursive Feature Elimination (RFE) Approach

Ahmad Fikri Zulfikar (University of Pamulang \& Universiry of Bina Nusantara, Indonesia)

Comparison of Modulation Schemes toward Coverage Area in indoor Visible Light Communication

Andrik Supadiyanto (Telkom University, Indonesia), Brian Pamukti (Telkom University, Indonesia), Desti Madya Saputri (Telkom University, Indonesia), Nur Andini (Telkom University, Indonesia)

Parallel Session 2-E

Speech recognition for Indonesian language and its application to home automation

Zulkarnaen Hatala (Politeknik Negeri Ambon, Indonesia)

Aspect and Opinion Word Extraction on Opinion Sentences in Bahasa Indonesia using Rule Based Generated from Regular Expression Yuliana Setiowati (Politeknik Elektronika Negeri Surabaya, Indonesia), Fitri Setyorini (Politeknik Elektronika Negeri Surabaya, Indonesia), Afrida Helen (Universitas Padjadjaran (Unpad), Indonesia)

The Use Matriks of Linear and Quadratic Regression to Predict Number Electricity Distributed in Indonesia

Desty Rakhmawati (Universitas Amikom Purwokerto, Indonesia), Hendra Marcos (STMIK AMIKOM Purwokerto, Indonesia), Utami Puspita (Universitas AMIKOM Purwokerto, Indonesia), Uswatun Hasanah (STMIK Amikom Purwokerto, Indonesia)

An Optimization of a Lexicon Based Sentiment Analysis Method on Indonesian App Review

Bayu Trisna Pratama (Universitas Amikom Yogyakarta, Indonesia), Ema Utami (Universitas Amikom Yogyakarta, Indonesia), Andi Sunyoto (Universitas AMIKOM Yogyakarta, Indonesia)

Classification of Citation Sentence for Filtering Scientific References

Ghoziyah Haitan Rachman (Institut Teknologi Bandung, Indonesia), Masayu Leylia Khodra (Institut Teknologi Bandung, Indonesia), Dwi H Widyantoro (Institut Teknologi Bandung, Indonesia)

Indonesian Part of Speech Tagging Using Hidden Markov Model - Ngram \& Viterbi

Denis Eka Cahyani (Universitas Sebelas Maret, Indonesia)

Mining Student Feedback to Improve the Quality of Higher Education through Multi Label Classification, Sentiment Analysis, and Trend Topic Calandra Alencia Haryani (Universitas Indonesia \& Universitas Pelita Harapan, Indonesia), Achmad Hidayanto (University of Indonesia, Indonesia), Nur Fitriah Ayuning Budi (Universitas Indonesia, Indonesia), Zaenal Abidin (Universitas Negeri Semarang, Indonesia), Theresia Wati (Universitas Pembangunan Nasional Veteran Jakarta, Indonesia)

Mobile Business Intelligence Assistant ( $m$-BELA) for Higher Education Executives

Mohamad Irwan Afandi (Universitas Pembangunan Nasional Veteran Jawa Timur, Indonesia), Eka Dyar Wahyuni (Universitas Pembangunan Nasional Veteran Jawa Timur, Indonesia), Siti Mukaromah (Universitas Pembangunan Nasional Veteran Jawa Timur, Indonesia)

Parallel Session 3-A

Design of PID Controllers for Speed Control of Three Phase Induction Motor Based on Direct-Axis Current (Id) Coordinate Using IFOC Indra Ferdiansyah (Politeknik Elektronika Negeri Surabaya, Indonesia), Diah Yanaratri (Politeknik Elektronika Negeri Surabaya, Indonesia), Lucky Pradigta Setiya Raharja (Politeknik Elektronika Negeri Surabaya, Indonesia), Era Purwanto (Electronic Engineering Polytechnic Institute Of Surabaya, Indonesia) 
Dynamic Source Routing and Optimized Link State Routing Performance in Multipath Fading Environment with Dynamic Network Topology Istikmal Istikmal (Telkom University, Indonesia), Agus Subekti (Indonesian Institute of Sciences, Indonesia), Doan Perdana (Telkom University, Indonesia), Ridha Negara (Telkom University, Indonesia), Arif Indra Irawan (Telkom University, Indonesia), Sussi Sussi (Telkom University, Indonesia)

Comparative Analysis of Codec G.729 and G.711 on IEEE 802.11AH with MCS and Raw Slot Change Mechanism for VOIP Service Doan Perdana (Telkom University, Indonesia), Istikmal Istikmal (Telkom University, Indonesia)

Flooding Detection System Based on Water Monitoring and ZigBee Mesh Protocol

Herman Yuliandoko (State Polytehnic of Banyuwangi, Indonesia), Abdul Rohman (State Polytechnic of Banyuwangi, Indonesia)

Performance Comparison of Blackman, Bartlett, Hanning, and Kaiser Window for Radar Digital Signal Processing

Sulis Tyaningsih (Indonesian Institute of Science (LIPI), Indonesia), Prasetyo Putranto (Indonesian Institute of Sciences, Indonesia),

Taufiqqurrachman Taufiq (National Taiwan University of Science and Technology \& Indonesian Institute of Sciences, Indonesia), Winy Desvasari (Indonesian Institute of Sciences, Indonesia), Yusuf Nur Wijayanto (Indonesian Institute of Sciences (LIPI), Indonesia),

Pamungkas Daud (Indonesian Institute of Sciences, Indonesia), Dadin Mahmudin (Indonesian Institute of Sciences (LIPI), Indonesia), Deni

Permana Kurniadi (LIPI, Indonesia), Arief Rahman (Indonesian Institute of Sciences, Indonesia), Sri Hardiati (Indonesian Institute of Science (LIPI), Indonesia), Arie Setiawan (Indonesian Institute of Sciences, Indonesia), Fajri Darwis (Indonesian Institute of Sciences (LIPI),

Indonesia), Eko Pristianto (Indonesian Institute of Sciences, Indonesia)

Implementation of Algorithm Rabin-Karp for Thematic Determination of Thesis

Ainul Yaqin (Universitas Amikom Yogyakarta, Indonesia), Akhmad Dahlan (Universitas Amikom Yogyakarta, Indonesia), Reno Diandika Hermawan (University of Amikom, Indonesia)

Hybrid Resampling for Imbalanced Class Handling on Web Phishing Classification Dataset

Yoga Pristyanto (Universitas Amikom Yogyakarta, Indonesia), Akhmad Dahlan (Universitas Amikom Yogyakarta, Indonesia)

Parallel Session 3-B

Modeling of Time Series Data Prediction using Fruit Fly Optimization Algorithm and Triple Exponential Smoothing

Ryan Putranda Kristianto (Universitas Amikom Yogyakarta, Indonesia)

A Novel Ant Colony Optimization Algorithm for Waste Collection Problem

Sarifah Putri Raflesia (Universitas Sriwijaya \& Institut Teknologi Bandung, Indonesia), Anugrah K Pamosoaji (Universitas Atma Jaya

Yogyakarta, Indonesia)

Fuzzy Hierarchical Model and Particle Swarm Optimization in Gas Leakage Detector Mobile Robot

Kanda Januar Miraswan (Universitas Sriwijaya, Indonesia), Muhammad Ali Buchari (Universitas Sriwijaya, Indonesia), Rizki Kurniati

(Universitas Sriwijaya, Indonesia)

Optimization of Weight Backpropagation with Particle Swarm Optimization for Student Dropout Prediction

Eka Yulia Sari (Universitas Amikom Yogyakarta, Indonesia), Kusrini Kusrini (AMIKOM Yogyakarta University, Indonesia), Andi Sunyoto (Universitas AMIKOM Yogyakarta, Indonesia)

The Mapping of Lighting Intensity from the Light Distribution on LED and CFL Lamps

Herlina Wahab (Sriwijaya University \& University of Indonesia, Indonesia), Rudy Setiabudy (Universitas Indonesia (UI), Indonesia)

Impact of STATCOM Installation on Power System's Voltage Stability Performance

Nurriza Kholifatulloh Hasanah (Universitas Gadjah Mada, Indonesia), Lesnanto Multa Putranto (UGM, Indonesia), Sasongko Hadi (Universitas Gadjah Mada, Indonesia), Febian Melwa Reksa Aditya (Universitas Gadjah Mada, Indonesia)

Analysis Of Overclock Ram Galax Hall Of Fame For Daily Needs

Rizqi Sukma Kharisma (Universitas AMIKOM Yogyakarta, Indonesia), Miko Kastomo Putro (Universitas AMIKOM Yogyakarta, Indonesia)

\section{Parallel Session 3-C}

Analysis of Review And Rating on Consumer Trust in Jakarta Taking Online Booking Queue Based on Tam Hendrico Andre (Bina Nusantara University, Indonesia), Sfenrianto Sfenrianto (Bina Nusantara University, Indonesia), Gunawan Wang (Bina Nusantara University, Indonesia), Pangondian Prederikus (Bina Nusantara, Indonesia)

Analysis of the Effect of Security and Trust on Buying Decision On the Tokopedia Mobile Apps

Edward Chandra (Bina Nusantara University, Indonesia), Stefanie Liu (Bina Nusantara University, Indonesia), Sfenrianto Sfenrianto (Bina Nusantara University, Indonesia), Gunawan Wang (Bina Nusantara University, Indonesia)

Behavior Intention of Information Technology Students in Using Youtube as Learning Resources

Paul Weniko (Bina Nusantara University, Indonesia), Gunawan Wang (Bina Nusantara University, Indonesia), Sfenrianto Sfenrianto (Bina Nusantara University, Indonesia), Muhammad Aldenny (Bina Nusantara University, Indonesia)

Analysis of the Effect of Trust on Purchase Intention in E-Commerce Integration for Vendors \& Event Organizer

Dina Ikramina (Bina Nusantara University, Indonesia), Sfenrianto Sfenrianto (Bina Nusantara University, Indonesia), Gunawan Wang (Bina Nusantara University, Indonesia)

Evaluating the Usability of Heuristics within Telegram using the Linear Regression Method

Rumini Rumini (Universitas AMIKOM Yogyakarta, Indonesia) 
Social Network Users Switching Platforms Behaviour: A Proposal for Research Explorations using a Mixed Method Approach Timothy McBush Hiele (Ming Chuan University, Taiwan), Andree E. Widjaja (Universitas Pelita Harapan, Indonesia), Calandra A. Haryani (Universitas Pelita Harapan, Tangerang, Indonesia)

Fingerprint Presence Fraud Detection Using Tight Clustering on Employee's Presence and Activity Data

Irfan Kamil (Institut Teknologi Bandung, Indonesia), Bambang Pharmasetiawan (Institut Teknologi Bandung, Indonesia)

\section{Parallel Session 3-D}

Analysis of Dominants Game Elements using the Sillaots Parameters and Octalysis Framework on the Google Play Store

Dema Mathias Lumban Tobing (Universitas Amikom Yogyakarta, Indonesia), Emma Utami (Universitas AMIKOM Yogyakarta, Indonesia), Hanif Fatta (Universitas AMIKOM Yogyakarta, Indonesia)

Pregnancy Mapping and Monitoring Web Based Geographic's Information System

Arief Munandar (Universitas AMIKOM Yogyakarta, Indonesia), Arief Setyanto (Universitas AMIKOM Yogyakarta, Indonesia), Suwanto Raharjo (Informatics of The Institut Sains \& Teknologi AKPRIND Yogyakarta, Indonesia), Gunawan Wicahyono (Amikom University Yogyakarta, Indonesia)

Integration of K-Means Clustering and Naive Bayes Classification Algorithms for Smart AC Monitoring and Control in WSAN

Ryan Putranda Kristianto (Universitas Amikom Yogyakarta, Indonesia), Banu Santoso (Universitas AMIKOM Yogyakarta, Indonesia)

Optimization of Transmission Expansion Planning Considering the System Losses: A Case Study of the Garver's 6-Bus System Afif Amalul Arifidin (Universitas Gadjah Mada, Indonesia), Sasongko Hadi (Universitas Gadjah Mada, Indonesia), Lesnanto Multa Putranto (UGM, Indonesia), Muhammad Yasirroni (Universitas Gadjah Mada, Indonesia)

Real-Time Irradiance Estimation Based on Maximum Power Current of Photovoltaic

Moh Syaiful Imam (Politeknik Elektronika Negeri Surabaya (PENS), Indonesia), Eka Prasetyono (Politeknik Elektronika Negeri Surabaya, Indonesia), Epyk Sunarno (Politeknik Elektronika Negeri Surabaya, Indonesia)

What are Customers Really Need in Ride Hailing Applications?Signaling Electronic Service Quality via E-CRM Features

Tifanny Nabarian (Universitas Indonesia, Indonesia, Indonesia), Yudho Sucahyo (University of Indonesia, Indonesia), Arfive Gandhi (Universitas Indonesia, Indonesia), Yova Ruldeviyani (Universitas Indonesia, Indonesia)

Realization of Point Cloud Maps Using ROS \& Visual Sensor on Raspberry Pi 3 Based Mobile Robot

Husnairi Ardan Miranto (Telkom University, Indonesia), Agung Nugroho Jati (Telkom University, Indonesia), Casi Setianingsih (Telkom University, Indonesia)

Parallel Session 3-E

Essential Blockchain Technology Adoption factors in Pharmaceutical Industry

Surjandy Surjandy (Bina Nusantara University, Indonesia), Erick Fernando (Bina Nusantara University, Indonesia), Meyliana Meyliana (Bina Nusantara University, Indonesia)

Performance Evaluation of XPath Routing Protocol in Data Center Network using NS3-Simulator

Gerry Wowiling (Universitas Indonesia, Indonesia), Hermawan Rahman Sholeh (University of Indonesia, Indonesia), Ruki Harwahyu (Universitas Indonesia \& Universitas Indonesia, Indonesia), Riri Fitri Sari (University of Indonesia, Indonesia)

Job Seeker Profile Classification of Twitter Data Using the Naïve Bayes Classifier Algorithm Based on the DISC Method

Anggit Dwi Hartanto (Universitas Amikom Yogyakarta, Indonesia), Ema Utami (Universitas Amikom Yogyakarta, Indonesia), Sumarni Adi (Universitas AMIKOM Yogyakarta, Indonesia), Harish Setyo Hudnanto (Universitas AMIKOM Yogyakarta, Indonesia)

The Impact Of Features Selection On Performance Of Artificial Neural Network In Diagnosis Of Diabetic Retinopathy

Tri Astuti (Universitas Amikom Purwokerto, Indonesia), Rizki Wahyudi (STMIK Amikom Purwokerto, Indonesia), Uswatun Hasanah (STMIK Amikom Purwokerto, Indonesia), Bambang Pilu Hartato (STMIK AMIKOM Purwokerto, Indonesia), Zanuar Rifa'i (Stmik Amikom Purwokerto, Indonesia)

RS Code and Compressive Sampling on Video Watermarking-based DWT-SVD

Ledya Novamizanti (Telkom University, Indonesia)

Trending Topic Classification for Single-Label Using Multinomial Naive Bayes (MNB) and Multi-Label Using K-Nearest Neighbors (KNN) Denis Eka Cahyani (Universitas Sebelas Maret, Indonesia)

Influence of Voltage System-Level to the dimensions and performance of Squirrel Cage Induction Motor Three Phase 50Hz $5 \mathrm{HP}$ for Electric Vehicles

Danang Wijaya (UGM, Indonesia), Iftitah Imawati (Universitas Gadjah Mada, Indonesia) 


\section{Comparison of Classification Methods using}

\section{Historical Loan Application Data}

\author{
Yohanes R. Laberto Kelen \\ laberto.kelen@gmail.com
}

Magister Informatika, Universitas Atma Jaya Yogyakarta ${ }^{1}$

Indonesia 55281

\author{
Andi Wahju Rahardjo Emanuel \\ andi.emanuel@uajy.ac.id \\ Magister Informatika,Universitas Atma Jaya Yogyakarta \\ Indonesia 55281
}

\begin{abstract}
Every year, the number of cooperatives in the province of East Nusa Tenggara continues to grow. Cooperatives are present with the aim of helping the community on the financial side. The cooperative offers the principle of saving and providing low-interest loans to its members. But there are times when lending is subjective. This condition is a major factor in the occurrence of errors in providing credit that leads to congestion (non-performing loans). This study focuses on the comparison of five classification methods using historical loan application data for a Multipurpose Cooperative in East Nusa Tenggara. The 5 methods are Naïve Bayes, K-Nearest Neighbor (KNN), Support Vector Machine (SVM) Random Forest, and C4.5. In the test results, it turns out that the $\mathbf{C} 4.5$ Method has better accuracy and a smaller error rate.
\end{abstract}

Keywords-big data, classification, cooperative, Historical Loan Application.

\section{INTRODUCTION}

Based on article 4 in Indonesian Law Number 17 of 2012 concerning cooperatives, cooperatives have a goal to prosper members in particular and society in general [1]. The cooperative in the island province of East Nusa Tenggara is expected to be the leading spearhead in the economy in the community.

The development of cooperatives in East Nusa Tenggara continues to increase. The number of cooperatives in this province in 2018 was 4,317 units. There was a significant increase in the number compared to 2014, which amounted to 2,818 active cooperative units. Cooperatives help the community of East Nusa Tenggara because it is considered very easy to reach and not difficult for the community to get funds. The interest offered by the cooperative is so small that the community is interested in joining the Cooperative.

But at a Multipurpose Cooperative in East Nusa Tenggara, researchers found cases of Non-Performing Loan (NPL). NPL is the failure of customers to repay loans. It turns out that this case often occurs in cooperatives, as seen from the percentage of NPL from 2014-2017, which reached $76 \%$. It turned out that the consideration of lending, which was declared feasible by the creditor was sometimes subjective. Loans are given to members because there are elements of kinship or acquaintances without looking at the loan criteria themselves. This is also a major factor in the cause of non-performing loans.
In this study, five classification methods will be compared using historical loan application data. The loan application data consists of 6 criteria, namely the loan amount, collateral amount, loan period, collateral status, type of interest, and marital status. The contribution of the proposed research is finding the best method of accuracy, so it can be used as a reference for further research, such as to develop a knowledge base and decision support system. So that going forward, the process of granting credit to customers can pay attention to these available criteria.

\section{RELATED WORKS}

The following are some previous studies on the classification methods that will be discussed in this paper. Naive Bayes Classifier is a popular algorithm that can be used to predict singers just by lyrics. Using available datasets, this method successfully predicts with very good results [2]. Naïve Bayes ability is used to classify SMS messages for Disaster Response. The test results get an accuracy of up to $89 \%$ [3]. Using the K-Nearest Neighbor classification approach, grape leaf disease is classified effectively. Although leaf images and identification of leaf disease are not easy tasks using images of agronomic plants [4].

Support Vector Machine (SVM) is the new machine learning algorithm used in pattern recognition problems including texture classification [5]. SVM minimizes structural risk, which excludes traditional empirical risk minimization, which is commonly used in conventional neural networks [5][6]. SVM is used to classify Bangla Language scripts with accuracy reaching an average of $94.78 \%$ [7]. Decision trees are algorithms that are very fast to be trained and evaluated. This method is also very easy to interpret and can be visualized [8]. Random forest and C4.5 are methods in the decision tree algorithm.

Random forest-based techniques can be used more useful for unsupervised learning [9]. C4.5 algorithm is a classification algorithm with a decision tree technique. C4.5 is the development of the ID3 algorithm [10]. This method is often used because accuracy tends to be high and has been used by many researchers for classification purposes [11]. 


\section{METHODOLOGY}

\section{A. Machine Learning Classifier}

In this paper, the author examines five different classification methods. Some of these algorithms will be explained in this section:

(1) Naïve Bayes is a classification supervised learning algorithm [12]. This is a popular algorithm based on conditional probability theorems to determine new feature vector classes.

(2) K-Nearest Neighbor (KNN) classification algorithm that is safe and efficient can hide class labels and data access patterns. Viewed from the length of the classification time, the results of the performance analysis of this method achieved 17 times better performance than the existing scheme [13].

(3) Support Vector Machine (SVM) is SVM is a wellknown classification technique based on theory statistical learning [14]. SVM consists of a two classes classification model; a model defined in the feature space of the largest linear classifier [15];

(4) Random forest is an algorithm which based techniques can be used more useful for unsupervised learning [9]. RF is very efficient with low computational costs, few parameter settings, and good performance [16].

(5) C4.5 algorithm is a classification algorithm with a decision tree technique [17]. C4.5 is the development of the ID3 algorithm [10]. This method is often used because accuracy tends to be high and has been used by many researchers to classify things [11].

\section{B. Data source}

The authors have conducted research at a Multipurpose Cooperative in East Nusa Tenggara. They have allowed the author to use their historical loan application data for 20162017. The number of data samples used is 2500 loan application data. The author uses a ratio of 80:20 for the test scenario. So, 2000 data is used for training data and the rest is used as test data.

This historical loan application data has been edited so that attributes are formed. Table 1 shows 6 attribute variables. The six attributes include loan amount (A1), collateral amount (A2), loan period (A3), collateral status (A4), loan interest (A5), and marital status (A6).

TABLE I. DISTRIBUTION OF ATTRIBUTES

\begin{tabular}{|l|l|}
\hline Code & Attribute Name \\
\hline A1 & Loan amount \\
\hline A2 & Collateral amount \\
\hline A3 & Loan period \\
\hline A4 & Collateral status \\
\hline A5 & Loan interest \\
\hline A6 & Marital status \\
\hline
\end{tabular}

\section{Discretization}

Discretization converts continuous data values into categorical data. In this paper, discretion is carried out on class attributes, namely the loan amount (A1), collateral amount (A2) and load period (A3) attributes.
TABLE II. LOAN AMOUNT CLASSIFICATION

\begin{tabular}{|c|c|c|}
\hline Class & Intervals (IDR) & Class Name \\
\hline 1 & $<2.000 .000$ & Very Low $(\mathrm{VL})$ \\
\hline 2 & $2.000 .000-4.000 .000$ & Low $(\mathrm{L})$ \\
\hline 3 & $4.000 .000-6.000 .000$ & Moderate $(\mathrm{M})$ \\
\hline 4 & $6.000 .000-8.000 .000$ & High $(\mathrm{H})$ \\
\hline 5 & $>8.000 .000$ & Very High $(\mathrm{VH})$ \\
\hline
\end{tabular}

This classification is intended to label data. In table 2, it can be seen that there are 5 classes for the loan amount attribute (A1). The class is very low (VL), low (L), moderate $(\mathrm{M})$, high $(\mathrm{H})$ and very high $(\mathrm{VH})$.

TABLE III. COLLATERAL AMOUNT CLASSIFICATION

\begin{tabular}{|c|c|c|}
\hline Class & Intervals (IDR) & Class Name \\
\hline 1 & $<2.000 .000$ & Low $(\mathrm{L})$ \\
\hline 2 & $2.000 .000-4.000 .000$ & Moderate $(\mathrm{M})$ \\
\hline 3 & $>4.000 .000$ & High $(\mathrm{H})$ \\
\hline
\end{tabular}

Class for loan value (A2) consists of low (L), moderate $(\mathrm{M})$, and high $(\mathrm{H})$ can be seen in table 3. The amount of collateral is one of the conditions in making a loan. collateral is usually seen from the number of members' deposits in the cooperative. But it can also be in the form of proof of ownership of valuables. in this study, collateral is used in the form of cooperative deposits.

TABLE IV. LOAN PERIOD CLASSIFICATION

\begin{tabular}{|c|c|c|}
\hline Class & Intervals & Class Name \\
\hline 1 & 6 months & Very slow (VS) \\
\hline 2 & 10 months & Slow (S) \\
\hline 3 & 12 months & Moderate (M) \\
\hline 4 & 18 months & Fast (F) \\
\hline 5 & 24 months & Very fast (VF) \\
\hline
\end{tabular}

Class for loan period (A5) can be seen in table 4. the five classes are very slow (VS), slow (S), moderate (M), fast (F), and very fast (VF). The loan period is the time needed by the cooperative members to repay the loan to the cooperative.

All attribute elements have several categories which can be seen in table 5 .

TABLE V. DISTRIBUTION OF CLASS

\begin{tabular}{|l|l|}
\hline $\begin{array}{l}\text { Attribute } \\
\text { Code }\end{array}$ & Class Name (Class Code) \\
\hline A1 & $\begin{array}{l}\text { Very Low (VL), Low (L), Moderate (M), } \\
\text { High (H), Very High (VH) }\end{array}$ \\
\hline A2 & Low (L), Moderate (M), High (H) \\
\hline A3 & $\begin{array}{l}\text { Very slow (VS) Slow (S), Moderate (M), } \\
\text { Fast (F), Very fast (VF) }\end{array}$ \\
\hline A4 & True (T), False (F) \\
\hline A5 & Down interest (D), Flat interest (F) \\
\hline A6 & Married (M), Single (S) \\
\hline
\end{tabular}

After the steps above, the next step is to determine the attribute results (A8). Authors aiming at classification will classify two classes, Lancar and Macet. Lancar class declared for cooperative members who will successfully repay the loans; and Macet class if the loan will fails to pay off or non-performing loan (NPL). Testing the data will be explained in the next sub-chapter. 


\section{Evaluation}

The confusion matrix is used to find the accuracy of classification model in testing several methods in this study [10] [11]. The confusion matrix is a matrix formed by several training data and test data.

The confusion matrix size $\mathrm{n} \times \mathrm{n}$ shows the results of prediction and classification, where $\mathrm{n}$ is the number of predetermined class attributes. The four table fields contain the number of documents classified as True Positive (TP), True Negative (TN), False Positive (FP) and False Negative $(\mathrm{FN})$.

TABLE VI. CONFUSION MATRIX

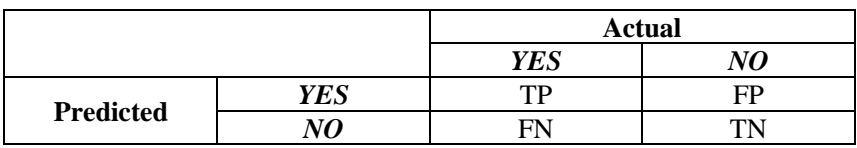

TP is the amount of positive data that is predicted right. $\mathrm{TN}$ is the amount of positive data that is predicted wrong. FP is the amount of negative data that is predicted right. TN is the amount of negative data that is predicted wrong.

Accuracy is the percentage of the results of the comparison of cases that were correctly identified by the number of all cases. The accuracy of the classifier can be found by formula 1 .

$$
\text { Accuracy }=\frac{T P+T N}{T P+T N+F P+F N}
$$

Precision, the positive predictive value between samples taken. The following formula calculates precision:

$$
\text { Precision }=\frac{T P}{T P+F P}
$$

APER: (Apparent Error Rate) or the so-called error rate is an evaluation measure that is used to see opportunities for classification errors produced by a classification function. The smaller the APER value, the better the classification results. The calculation of the APER can be seen in equation 3.

$$
A P E R=\frac{F P+F N}{T P+T N+F P+F N}
$$

\section{EXPERIMENTAL RESULTS}

The authors tested 500 test data on 2000 training data with WEKA data mining software that produced a pretty good percentage. The classification results are shown in table 7 .

TABLE VII. ClASSIFICATION TEST

\begin{tabular}{|c|c|c|c|}
\hline Methods & $\begin{array}{c}\text { Correctly } \\
\text { classified }\end{array}$ & $\begin{array}{c}\text { Incorrectly } \\
\text { classified }\end{array}$ & $\%$ \\
\hline NB & 491 & 9 & 98.2 \\
\hline KNN & 495 & 5 & 99 \\
\hline SVM & 493 & 7 & 98.6 \\
\hline RF & 495 & 5 & 99 \\
\hline C4.5 & 496 & 4 & 99.2 \\
\hline
\end{tabular}

C4.5 classifier algorithm gives the highest classification in terms of correctly classified instances [18]. Table 8 shows the results of the classification of the random forest method and KNN obtained a value of $99 \%$ and the highest classification obtained by method $\mathrm{C} 4.5$ with a value of $99.2 \%$.

TABLE VIII. CONFUSION MATRIX FROM TEST RESULT

\begin{tabular}{|c|c|c|c|c|}
\hline Methods & TP & TN & FP & FN \\
\hline NB & 328 & 0 & 9 & 163 \\
\hline KNN & 334 & 2 & 3 & 161 \\
\hline SVM & 332 & 2 & 5 & 161 \\
\hline RF & 334 & 2 & 3 & 161 \\
\hline C4.5 & 335 & 2 & 2 & 161 \\
\hline
\end{tabular}

Table 8 shows a confusion matrix classified by WEKA data mining software with class results (A8). Table 9 shows the comparison results. Each method tested has a value of True Positive and False Negative are greater than True Negative and False Positive. This proved that the success rate of classification correctly is very high according to table 7.

TABLE IX. COMPARISON OF RESULT

\begin{tabular}{|c|c|c|c|}
\hline Methods & Accuracy & Precision & APER \\
\hline NB & 98.2 & 100 & 1.80 \\
\hline KNN & 99 & 99.4 & 1.00 \\
\hline SVM & 98.6 & 99.4 & 1.40 \\
\hline RF & 99 & 99.4 & 1.00 \\
\hline C4.5 & 99.2 & 99.41 & 0.80 \\
\hline
\end{tabular}

The results of the testing experiment show that the $\mathrm{C} 4.5$ method has the smallest APER value with 0.80. C4.5 method also has the biggest accuracy with $99.2 \%$. Naïve Bayes has the biggest precision with $100 \%$.

The classification method $\mathrm{C} 4.5$ has a good result than the other four classification methods with a dataset for applying the credit to a cooperative in East Nusa Tenggara. The C4.5 method on WEKA Data Mining Software turns out to produce a decision tree. The decision tree can be converted into programming logic.

\section{TABLE $X . \quad$ KNOWLEDGE BASE}

$$
\begin{aligned}
& \text { If A } 1=\text { Very Low then Lancar } \\
& \text { If } \mathrm{A} 1=\text { Low then Lancar } \\
& \text { If } \mathrm{A} 1=\text { Moderate then Lancar } \\
& \text { If } \mathrm{A} 1=\text { High then } \\
& \text { If A6 = Married then Macet } \\
& \text { If A6 = Single then Lancar } \\
& \text { If A } 1=\text { Very High then } \\
& \text { If A6 = Married then Macet } \\
& \text { If A6 = Single then } \\
& \text { If A } 2=\text { Low then } \\
& \text { If A5 = Flat Interest then Macet } \\
& \text { If A5 = Down Interest then Lancar } \\
& \text { If A } 2=\text { High then Macet } \\
& \text { If A } 2=\text { Moderate then } \\
& \text { If A } 5=\text { Flat Interest then } \\
& \text { If A } 4=\text { True then Macet } \\
& \text { If A } 4=\text { False then Lancar } \\
& \text { If A5 = Down Interest then Macet }
\end{aligned}
$$

The logic in table 10 can be implemented into a knowledge base for a decision support system. 


\section{CONCLUSION AND DISCUSSION}

Each loan submitted by the members of the cooperative must be well considered by the creditor. Credit criteria are expected to be an important concern. Avoid accepting loan proposals based on subjective factors. This paper shows the results of the highest testing accuracy obtained by method C4.5 with a percentage of $99.41 \%$. Naïve Bayes has the best precision of the five tested methods.

The results are obtained from the calculation of the confusion matrix. The confusion matrix is generated from WEKA data mining software. Accuracy can be increased by adding training data, attributes. Besides that, you can also use algorithm optimization. The results of this paper are not only comparisons between the five classification methods. But there is a decision tree based on the $\mathrm{C} 4.5$ algorithm. This decision tree is expected to be a reference for creditors to determine loan proposals that fit the criteria.

\section{REFERENCES}

[1] "UNDANG-UNDANG REPUBLIK INDONESIA NOMOR 17 TAHUN 2012 TENTANG PERKOPERASIAN," pp. 1$56,2012$.

[2] D. Buži and J. Dobša, "Lyrics Classification using Naive Bayes," 2018 4lst Int. Conv. Inf. Commun. Technol. Electron. Microelectron., pp. 1011-1015, 2018.

[3] A. J. Ordoñez, R. E. J. Paje, and R. N. Naz, "SMS Classification Method for Disaster Response using Naïve Bayes Algorithm," pp. 233-236, 2018.

[4] I. Conference and C. Systems, "An Individual Grape Leaf Disease Identification Using Leaf Skeletons and KNN Classification," 2017.

[5] G. S. Raghtate, "Comparison of Classification Methods with Second Order Statistical Analysis and Wavelet Transform For Texture Image Classification," 2015.

[6] S. Begum, R. Sarkar, and A. Adaboost, "Cancer Classification from Gene Expression Based Microarray Data Using SVM Ensemble," pp. 13-16, 2015.
[7] M. K. Shukla, A. Rana, and H. Banka, "Classification of the Bangla Script Document using SVM,” pp. 31-34, 2016.

[8] S. H. Ahn et al., "Comparison of Clustering Methods for Obesity Classification," 2018 IEEE Int. Conf. Ind. Eng. Eng. Manag., pp. 1821-1825, 2018.

[9] S. Hussain, S. Muhammad, and M. Majid, "Segmentation of glioma tumors in brain using deep convolutional neural network," in Neurocomputing, 2018, vol. 282, pp. 248-261.

[10] C. Algorithm and J. A. Suyatno, "Rainfall Forecasting in Bandung Regency using," 2018 6th Int. Conf. Inf. Commun. Technol., vol. 0, no. c, pp. 324-328, 2018.

[11] A. G. Pertiwi, T. Widyaningtyas, and U. Pujianto, "Classification of Province Based on Dropout Rate," pp. 410-413, 2017.

[12] A. U. Haq, J. P. Li, M. H. Memon, S. Nazir, and R. Sun, “A Hybrid Intelligent System Framework for the Prediction of Heart Disease Using Machine Learning Algorithms," in Mobile Information Systems, 2018, vol. 2018.

[13] H. Kim and H. Kim, "A Privacy-Presering k NN Classification Algorithm using Yao's garbled circuit on Cloud Computing," 2017.

[14] H. Elaidi, Y. Elhaddar, Z. Benabbou, and H. Abbar, "An Idea of a Clustering Algorithm using Support Vector Machines Based on Binary Decision Tree," in 2018 International Conference on Intelligent Systems and Computer Vision, ISCV 2018, 2018, vol. 2018-May, no. 5, pp. 1-5.

[15] G. Cheng and X. Tong, "Fuzzy Clustering Multiple Kernel Support Vector Machine," in 2018 International Conference on Wavelet Analysis and Pattern Recognition, 2018, vol. 2018-July, pp. 7-12.

[16] H. Zhong, X. Song, and L. Yang, "Vessel Classification from Space-based AIS Data Using Random Forest," in 2019 5th International Conference on Big Data and Information Analytics (BigDIA), 2019, pp. 9-12.

[17] M. E. Cintra, M. C. Monard, and H. A. Camargo, "A Fuzzy Decision Tree Algorithm Based on C4.5," Mathware \& Soft. Computing Magazine, vol. 20, pp. 56-62.

[18] H. Erol, "Classification Performances Of Data Mining Clustering Algorithms For Remotely Sensed Multispectral Image Data," pp. 1-4, 2018. 


\section{CERTIFICATE}

This certificate is presented to

\section{Mr. Andi Wahju Rahardjo Eman-} uel

\section{as}

for outstanding contribution in the 4th 2019 International Conference on Information Technology, Information System, and Electrical Engineering held in Yogyakarta, Indonesia on 20 - 21 November 2019

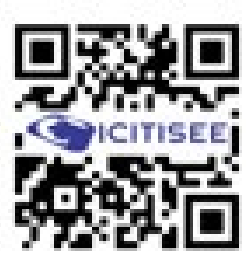

Conference No. \#48480

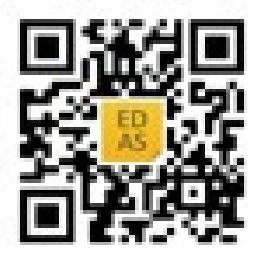

ID number 1129597

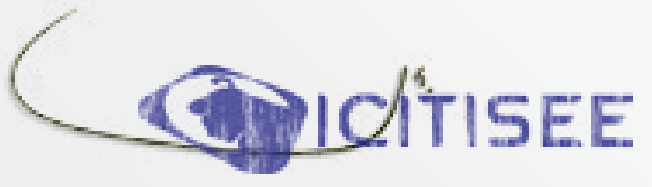

Dr. Kusrini, M.Kom.

General Chair

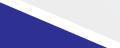

- IEEE INDONESIA SECTION
Department of mation Engineering Universitas Gadjah Mada 